\title{
Approximate Solution of Boundary Value Problems on Infinite Intervals by Collocation Methods*
}

\author{
By Christian Schmeiser
}

\begin{abstract}
The numerical solution of boundary value problems for ordinary differential equations on infinite intervals is considered. The infinite interval is cut at a finite, large enough point and additional boundary conditions are posed there. For the solution of the resulting problem, $A$-stable symmetric collocation methods are employed. Using the behavior of the solution of the "infinite" problem, meshes are defined which avoid an unreasonably high number of meshpoints. Stability and convergence of the resulting schemes are shown.
\end{abstract}

1. Introduction. In this paper we analyze numerical methods for the solution of problems of the form

$$
\begin{gathered}
y^{\prime}=f(t, y), \quad 0 \leqslant t<\infty, \\
\text { (1.1a) } \quad b(y(0))=0, \\
\text { (1.1c) } y \in C[0, \infty] \leftrightarrow\left\{y \in C[0, \infty), \lim _{t \rightarrow \infty} y(t) \text { exists and is finite }\right\},
\end{gathered}
$$

where $f, y \in \mathbf{R}^{n}$ and $b \in \mathbf{R}^{k}$ with $k \leqslant n$. The Jacobian $f_{y}(\infty, y(\infty))$ has no purely imaginary eigenvalues and there is a complete set of eigenvectors corresponding to the zero eigenvalue. Problems of this form occur, for example, in fluid dynamics and electronics. A vast number of applications result from an asymptotic analysis of singular perturbation problems where (1.1) defines boundary layer solutions.

The numerical solution proceeds in two steps. First, we apply the theory of Markowich [7] and replace (1.1) by the approximating "finite" problem

$$
\begin{gathered}
x_{T}^{\prime}=f\left(t, x_{T}\right), \quad 0 \leqslant t \leqslant T, \\
b\left(x_{T}(0)\right)=0, \\
S(T) x_{T}(T)=\sigma(T) .
\end{gathered}
$$

Thus, we consider the differential equation only on the finite interval $[0, T]$ and replace (1.1c) by the so-called asymptotic boundary condition (1.2c), which can be chosen such that

$$
\lim _{T \rightarrow \infty}\left\|y-x_{T}\right\|_{[0, T]}=0
$$

Received October 23, 1984.

1980 Mathematics Subject Classification. Primary 34B15, 34C05, 34D05, 34A10, 34A11.

Key words and phrases. Nonlinear boundary value problems, asymptotic properties, difference equations, stability of difference equations.

*This work was supported by "Österreichischer Fonds zur Förderung der wissenschaftlichen Forschung". 
The second step is the numerical solution of (1.2). The methods we consider are $A$-stable, symmetric collocation methods. The most powerful among these are collocation at Gaussian and Lobatto points, because of their superconvergence properties. The simplest members of these families are the Box-scheme and the trapezoidal rule, respectively. We do not include one-sided methods (e.g., the implicit Euler scheme) in our discussion. As we admit unstable solution components, instabilities might occur if the meshsizes are not kept small.

The condition of small meshsizes for stability does not apply for the symmetric schemes, which is of great importance, because it would lead to an unreasonably high number of meshpoints for large values of $T$. We will show that for a given accuracy an exponentially increasing meshsize sequence can be used if $y(t) \rightarrow y(\infty)$ exponentially. Assumptions on the problem which guarantee the validity of this condition will be stated.

This paper extends the results of Markowich and Ringhofer [8], who consider problems with a singularity of the second kind at $t=\infty$. Their treatment does not include the case of a zero eigenvalue of $f_{y}(\infty, y(\infty))$, and the methods they analyze satisfy the condition that meshpoints and collocation points do not coincide, which is not the case for Lobatto-points.

The idea of exponentially increasing meshsizes was originated in the analysis of numerical methods for linear singular perturbation problems (see Ascher and Weiss [1]). The results of Markowich and Ringhofer [8] simplify the analysis for certain nonlinear singular perturbation problems (see Ascher and Weiss [2]). Hopefully, it will be one application of our theory to provide a tool for the extension of these results. An outline of the paper follows. In Section 2 we state some analytic results for boundary value problems on "long" and infinite intervals (see Markowich [7]). The numerical methods to be used are defined in Section 3, and their stability for linear problems is shown in Section 4. In Section 5 we state and prove the convergence results for nonlinear problems.

2. Analytic Preliminaries. In this section we give an outline of the first step of the approximate solution of (1.1), i.e., we replace (1.1) by a problem on a finite interval. The results can also be found in Markowich [7] in much greater detail. We require problem (1.1) to have a locally unique, exponentially decaying solution. This is guaranteed by the following assumptions:

(A1) $\|f(t, y(\infty))\|=O\left(e^{-\kappa t}\right)$ for some $\kappa>0$.

(A2) For the Jacobian $f_{y}(\infty, y(\infty))$ the relation

$$
E^{-1} f_{y}(\infty, y(\infty)) E=\Lambda=\left(\begin{array}{lll}
\Lambda_{-} & & \\
& \Lambda_{+} & \\
& & 0
\end{array}\right)
$$

holds for some matrix $E$, where the eigenvalues of the $n_{-} \times n_{-}$matrix $\Lambda_{-}$have negative real parts and the eigenvalues of the $n_{+} \times n_{+}$matrix $\Lambda_{+}$have positive real parts; $n=n_{-}+n_{+}+n_{0}$.

(A3) Let (1.1) have a solution $y^{*}(t)$ and the linearization

$$
\omega^{\prime}=f_{y}\left(t, y^{*}\right) \omega, \quad b_{y}\left(y^{*}(0)\right) \omega=0, \quad \omega \in C[0, \infty]
$$


have only the trivial solution. This condition implies $b \in \mathbf{R}^{n_{-}+n_{0}}$, by the theory of Markowich for linear problems.

An approximating finite problem for (1.1) of the form (1.2) is completely defined by the matrix $S(T)$ and $\sigma(T)$. We denote a row decomposition of $E^{-1}$ corresponding to the blocks of $\Lambda$ by

$$
E^{-1}=\left(\begin{array}{c}
E_{-}^{-1} \\
E_{+}^{-1} \\
E_{0}^{-1}
\end{array}\right) .
$$

Choose $S(T)=E_{+}^{-1}$ and $\sigma(T)=S(T) y^{*}(\infty)$. Then the following convergence result holds.

THEOREM 2.1. Let conditions (A1)-(A3) hold. Let $S(T)$ and $\sigma(T)$ be defined as above and $\lambda_{\min }$ be defined by

$$
\lambda_{\min }=\min \{\kappa, \underline{\lambda}\}-\nu>0,
$$

where $\nu$ is a positive constant, $\kappa$ is taken from condition (A.1) and

$$
\underline{\lambda}=\min \left\{-\operatorname{Re} \lambda, \lambda \text { is an eigenvalue of } \Lambda_{-}\right\} .
$$

Then the problem (1.2) has a locally unique solution $x_{T}$ which satisfies

$$
\left\|y^{*}-x_{T}\right\|_{L^{\infty}[0, T]}=O\left(\exp \left(-\lambda_{\min } T\right)\right) .
$$

In order to keep the error at $O(\varepsilon)$ we choose

$$
T(\varepsilon)=\frac{1}{\lambda_{\min }} \ln \frac{1}{\varepsilon} .
$$

3. Symmetric Collocation Methods. We consider collocation methods using continuous piecewise polynomials. Given a boundary value problem

$$
\begin{gathered}
y^{\prime}=f(t, y), \quad a \leqslant t \leqslant b, \\
b(y(a), y(b))=0,
\end{gathered}
$$

we define a mesh

$$
a \leqslant t_{0}<t_{1}<\cdots<t_{N}=b, \quad h_{i}=t_{i+1}-t_{i}, \quad i=0, \ldots, N-1 .
$$

A $k$-stage collocation method is then completely defined by a set of points $0 \leqslant u_{1}<u_{2}<\cdots<u_{k} \leqslant 1$ by requiring the approximate solution $y_{h}$ to be in

$$
C[a, b] \cap\left(\Pi_{k}\left[t_{0}, t_{1}\right] \times \cdots \times \Pi_{k}\left[t_{N-1}, t_{N}\right]\right),
$$

to satisfy the boundary conditions, and to satisfy the differential equation at the collocation points $t_{i j}=t_{i}+u_{j} h_{i} ; i=0, \ldots, N-1 ; j=1, \ldots, k$ (see de Boor and Swartz [4], Weiss [9]). Here $\Pi_{k}[\alpha, \beta]$ denotes the space of polynomials of degree at most $k$ on the interval $[\alpha, \beta]$.

We consider the case where the points $u_{j}, j=1, \ldots, k$, are placed symmetrically about $\frac{1}{2}$. Thus, the methods of interest fall into two classes:

I. $u_{1}>0, u_{k}<1$. In particular, the Gauss schemes belong to this class.

II. $u_{1}=0, u_{k}=1$. The Lobatto schemes are the most accurate members of this class. 
The growth functions of these methods satisfy (see Wright [10])

$$
\begin{gathered}
|\gamma(z)| \leqslant 1 \quad \text { for } \operatorname{Re} z \leqslant 0 \\
\gamma(-z)=(\gamma(z))^{-1} \\
e^{z}-\gamma(z)=O\left(z^{p}\right),
\end{gathered}
$$

where $p=2 k$ for Gauss schemes and $p=2 k-2$ for Lobatto schemes. The relation (3.1a) implies $A$-stability. It is a well-known fact that the collocation schemes are equivalent to certain implicit Runge-Kutta methods (see Axelsson [3], Weiss [9]), and can be written as systems of difference equations

$$
\begin{gathered}
\frac{y_{i j}-y_{i}}{h_{i}}=\sum_{l=1}^{k} a_{j l} f\left(t_{i l}, y_{i l}\right), \quad i=0, \ldots, N-1, j=1, \ldots, k, \\
\frac{y_{i+1}-y_{i}}{h_{i}}=\sum_{l=1}^{k} b_{l} f\left(t_{i l}, y_{i l}\right), \quad i=0, \ldots, N-1, \\
b\left(y_{0}, y_{N}\right)=0,
\end{gathered}
$$

where $a_{j l}=\int_{0}^{u_{j}} L_{l}(s) d s, b_{l}=\int_{0}^{1} L_{l}(s) d s$, the $L_{l}$ being the Lagrange polynomials for the $u_{j}, j=1, \ldots, k$. For a method of class II, $y_{i}=y_{i 1}=y_{i-1, k}$ holds, which implies that in the above system (3.2b) can be omitted. We set

$$
A=\left(a_{j l}\right), \quad j=1(1) k, l=1(1) k, \quad b=\left(b_{1}, \ldots, b_{k}\right)^{T} .
$$

Then Lemma 3.1 in Ascher and Weiss [1] implies that $A$ is invertible for methods of class I, and

$$
A=\left(\begin{array}{cc}
0 & 0 \\
\bar{a} & \bar{A}
\end{array}\right)
$$

for methods of class II, where the $(k-1) \times(k-1)$-matrix $\bar{A}$ is nonsingular. In order to state more properties of symmetric schemes, we introduce the operator $S$ acting on the space of matrices and being defined by

$$
S(X)=\left(x_{m-i+1, n-j+1}\right)_{i=1, \ldots, m ; j=1, \ldots, n} \text { for } X=\left(x_{i j}\right)_{i=1, \ldots, m ; j=1, \ldots, n} .
$$

By elementary calculations we obtain

$$
\begin{gathered}
S(I)=I, \quad S(\alpha X+\beta Y)=\alpha S(X)+\beta S(Y), \\
S(X Y)=S(X) S(Y), \quad S\left(X^{-1}\right)=[S(X)]^{-1}, \quad S^{-1}=S .
\end{gathered}
$$

We can now show

Lemma 3.1. (a) $A+S(A)=1_{k} b^{T}$ with $1_{k}=(1, \ldots, 1)^{T}$, (b) $S\left(b^{T}\right)=b^{T}$ hold for symmetric collocation schemes.

Proof. For symmetric schemes $u_{i}+u_{k-i+1}=1 ; i=1, \ldots, k$, holds. This implies for the Lagrange polynomials

$$
L_{i}(u)=L_{k-i+1}(1-u) .
$$

Using (3.4) we can show (a) by

$$
\begin{aligned}
a_{i j}+ & a_{k-i+1, k-j+1}=\int_{0}^{u_{i}} L_{j}(u) d u+\int_{0}^{u_{k-i+1}} L_{k-j+1}(u) d u \\
& =\int_{0}^{u_{i}} L_{j}(u) d u+\int_{0}^{1-u_{i}} L_{j}(1-u) d u=\int_{0}^{1} L_{j}(u) d u=b_{j}, \quad i, j=1, \ldots, k .
\end{aligned}
$$


Equation (3.4) immediately implies $b_{i}=b_{k-i+1}, i=1, \ldots, k$, which proves (b) and thus completes the proof.

\section{Stability for Linear Problems.}

(a) The Scalar Case. We start our treatment with the simple case of a scalar equation

$$
\begin{gathered}
y^{\prime}=\lambda y+f(t), \quad 0 \leqslant t \leqslant T, \\
y(0)=\bar{y},
\end{gathered}
$$

where $\lambda \in \mathbf{C}$, with $\operatorname{Re} \lambda<0$, and $f \in C[0, T]$. A collocation scheme for (4.1) has the form

$$
\begin{aligned}
& \hat{y}_{i}=1_{k} y_{i}+\lambda h_{i} A \hat{y}_{i}+h_{i} A \hat{f}_{i}, \\
& y_{i+1}=y_{i}+\lambda h_{i} b^{T} \hat{y}_{i}+h_{i} b^{T} \hat{f}_{i},
\end{aligned} \quad i=0, \ldots, N-1,
$$

$$
y_{0}=\bar{y} \text {, }
$$

where $\hat{y}_{i}=\left(y_{i 1}, \ldots, y_{i k}\right)^{T}$ and $\hat{f}_{i}=\left(f\left(t_{i 1}\right), \ldots, f\left(t_{i k}\right)\right)^{T}$. From (4.2a), $\hat{y}_{i}$ can be determined as

$$
\hat{y}_{i}=\left(I_{k}-\lambda h_{i} A\right)^{-1} 1_{k} y_{i}+\left(I_{k}-\lambda h_{i} A\right)^{-1} h_{i} A \hat{f}_{i},
$$

which yields

$$
y_{i+1}=\gamma\left(\lambda h_{i}\right) y_{i}+h_{i} \Gamma\left(\lambda h_{i}\right) \hat{f}_{i}, \quad y_{0}=\bar{y}
$$

where

$$
\begin{gathered}
\gamma(z)=1+z b^{T}\left(I_{k}-z A\right)^{-1} 1_{k}, \\
\Gamma(z)=b^{T}\left(I_{k}-z A\right)^{-1}
\end{gathered}
$$

for methods of class I, and

$$
\begin{gathered}
\gamma(z)=\left[\left(I_{k-1}-z \bar{A}\right)^{-1}\left(1_{k-1}+z \bar{a}\right)\right]_{k-1}, \\
\Gamma(z)=\left[\left(I_{k-1}-z \vec{A}\right)^{-1}(\bar{a}, \bar{A})\right]_{k-1}
\end{gathered}
$$

for methods of class II, where we used the last line in (4.3) and the form of $\left(I_{k}-z A\right)^{-1}$ stated in the proof of Lemma 4.1 below. For a representation of the solution of the homogeneous equation in (4.4), and a particular solution, we introduce the operators

$$
\begin{gathered}
Y_{n, m}(\lambda, h)=\prod_{j=n}^{m-1} \gamma\left(\lambda h_{j}\right), \\
H_{n, m}^{-}(\lambda, h) \hat{f}=\sum_{j=n}^{m-1} h_{j} Y_{j+1, m}(\lambda, h) \Gamma\left(\lambda h_{j}\right) \hat{f}_{j},
\end{gathered}
$$

where $h$ contains the information about the mesh and $\hat{f}^{T}=\left(\hat{f}_{0}^{T}, \ldots, \hat{f}_{n-1}^{T}\right)$. Using these definitions, the solution of (4.4) reads

$$
y_{i}=Y_{0, i}(\lambda, h) \bar{y}+H_{0, i}^{-}(\lambda, h) \hat{f}, \quad i=1,2, \ldots, N .
$$

In order to estimate the particular solution in (4.8), we need the following

Lemma 4.1. For $\operatorname{Re} z \leqslant 0$

$$
\left\|\left(I_{k}-z A\right)^{-1}\right\|_{\infty} \leqslant \text { const }
$$

holds, where const only depends on $A$. 
Proof. $A$-stability implies that

$$
\left(I_{k}-z A\right)^{-1} \text { exists for } \operatorname{Re} z \leqslant 0 .
$$

For methods of class I we write

$$
\left(I_{k}-z A\right)^{-1}=\frac{1}{z}\left(\frac{1}{z} I_{k}-A\right)^{-1} .
$$

We now use the invertibility of $A$ and obtain for $\left\|A^{-1}\right\| /|z| \leqslant \frac{1}{2}$, or $|z| \geqslant 2\left\|A^{-1}\right\|$, the estimate

$$
\left\|\left(I_{k}-z A\right)^{-1}\right\| \leqslant \frac{1}{|z|} 2\left\|A^{-1}\right\| \leqslant 1 .
$$

On the compact set $\left\{z \in \mathbf{C}|\operatorname{Re} z \leqslant 0| z \mid, \leqslant 2\left\|A^{-1}\right\|\right\}$, (4.9) obviously holds because of (4.10). For methods of class II, we similarly obtain

$$
\begin{gathered}
\left\|\left(I_{k-1}-z \vec{A}\right)^{-1}\right\| \leqslant \text { const } \text { for } \operatorname{Re} z \leqslant 0, \\
\left\|z\left(I_{k-1}-z \vec{A}\right)^{-1}\right\| \leqslant \text { const } \text { for } \operatorname{Re} z \leqslant 0
\end{gathered}
$$

and use

$$
\left(I_{k}-z A\right)^{-1}=\left(\begin{array}{c:c}
1 & 0 \\
\hdashline-z \bar{a} & I_{k-1}-z \bar{A}
\end{array}\right)^{-1}=\left(\begin{array}{c:c}
1 & 0 \\
\hdashline\left(I_{k-1}-z \vec{A}\right)^{-1} z \bar{a} & \left(I_{k-1}-z \vec{A}\right)^{-1}
\end{array}\right)
$$

which concludes the proof of Lemma 4.1.

In order to give a stability estimate for the solution of (4.2) we have to introduce norms. For $\hat{y}^{T}=\left(y_{0}, \hat{y}_{0}^{T}, y_{1}, \ldots, y_{N-1}, \hat{y}_{N-1}^{T}, y_{N}\right)$, we choose a discrete $L^{\infty}$-norm defined by

$$
\|\hat{y}\|_{L_{h}^{\infty}\left[t_{i}, t_{j}\right]}:=\max _{i \leqslant p \leqslant j-1} \max \left\{\left|y_{p}\right|,\left|y_{p+1}\right|,\left\|\hat{y}_{p}\right\|\right\},
$$

and for the inhomogeneity $\hat{f}$, the following discrete $L^{1}$-norm is used:

$$
\|\hat{f}\|_{L_{h}^{1}\left[t_{i}, t_{j}\right]}:=\sum_{l=i}^{j-1} h_{l}\left\|\hat{f}_{l}\right\|
$$

We can now state

LeMma 4.2. For the solution $\hat{y}$ of (4.2),

$$
\|\hat{y}\|_{L_{h}^{\infty}\left[t_{0}, t_{i}\right]} \leqslant \operatorname{const}\left(|\bar{y}|+\|\hat{f}\|_{L_{h}^{1}\left[t_{0}, t_{i}\right]}\right)
$$

holds.

Proof. We have

$$
\left|Y_{n, m}(\lambda, h)\right| \leqslant 1, \quad 0 \leqslant n<m \leqslant N
$$

by (3.1a), and

$$
\left\|\Gamma\left(\lambda h_{j}\right)\right\|_{\infty} \leqslant \text { const, } \quad 0 \leqslant j \leqslant N-1,
$$

by Lemma 4.1. Thus, (4.11) follows from (4.7), (4.8) and (4.3).

The case of a scalar differential equation with exponentially increasing solutions is treated similarly. 
We consider

$$
\begin{gathered}
y^{\prime}=\lambda y+f(t), \quad 0 \leqslant t \leqslant T, \\
y(T)=\bar{y},
\end{gathered}
$$

where $\lambda \in \mathbf{C}$, with $\operatorname{Re} \lambda>0$, and $f \in C[0, T]$. Using (3.1b) and (4.4), a collocation method for (4.12) can be written as

$$
y_{i}=\gamma\left(-\lambda h_{i}\right) y_{i+1}-h_{i} S\left(\Gamma\left(-\lambda h_{i}\right)\right) \hat{f}_{i}, \quad y_{N}=\bar{y},
$$

which follows from (3.3), (4.5) and Lemma 3.1 by virtue of

$$
\begin{aligned}
\gamma(-z) \Gamma(z) & =S(\gamma(-z)) \Gamma(z)=\left[1-z b^{T}\left(I_{k}+z S(A)\right)^{-1} 1^{k}\right] b^{T}\left(I_{k}-z A\right)^{-1} \\
& =b^{T}\left(I_{k}+z S(A)\right)^{-1}\left[I_{k}+z S(A)-z 1_{k} b^{T}\right]\left(I_{k}-z A\right)^{-1} \\
& =S\left[b^{T}\left(I_{k}+z A\right)^{-1}\right] .
\end{aligned}
$$

Formally, we can use the formulas (4.5) instead of (4.6) also for methods of class II, which makes the above proof applicable for this case, too. Solving the recursion (4.13) yields

$$
y_{i}=Y_{i N}(-\lambda, h) \bar{y}+H_{i, N}^{+}(\lambda, h) \hat{f}, \quad i=0,1, \ldots, \dot{N}-1,
$$

where $Y_{n, m}$ is defined in (4.7a) and

$$
H_{n, m}^{+}(\lambda, h) \hat{f}=-\sum_{j=n}^{m-1} h_{j} Y_{i, j}(-\lambda, h) S\left(\Gamma\left(-\lambda h_{j}\right)\right) \hat{f}_{j} .
$$

Analogously to Lemma 4.2, we obtain the stability result

$$
\|\hat{y}\|_{L_{h}^{\infty}\left[t_{i}, t_{N}\right]} \leqslant \operatorname{const}\left(|\bar{y}|+\|\hat{f}\|_{L_{h}^{1}\left[t_{i}, t_{N}\right]}\right) .
$$

Finally, we consider the case $\lambda=0$,

$$
y^{\prime}=f(t), \quad 0 \leqslant t \leqslant T
$$

$$
y(0)=\bar{y},
$$

where $f \in C[0, T]$.

Obviously, the solution of a collocation method is

$$
y_{i}=\bar{y}+H_{0, i}^{0}(h) \hat{f}
$$

where

$$
H_{n, m}^{0}(h) \hat{f}=-\sum_{j=n}^{m-1} h_{j} b^{T} \hat{f}_{j} .
$$

Clearly, the estimate (4.11) also holds for the solution of (4.18).

(b) Systems With Constant Coefficients. We consider problems of the form

$$
\begin{gathered}
y^{\prime}=M y+f(t), \\
B y(0)=\beta, \\
E_{+}^{-1} y(T)=\gamma,
\end{gathered}
$$

where $y$ is an $n$-vector and the $n \times n$-matrix $M$ can be transformed to block diagonal form by

$$
E^{-1} M E=\Lambda=\left(\begin{array}{lll}
\Lambda_{-} & & \\
& \Lambda_{+} & \\
& & 0
\end{array}\right)
$$


where the $n_{-} \times n_{-}$-matrix $\Lambda_{-}$has only eigenvalues with negative real parts and the $n_{+} \times n_{+}$-matrix $\Lambda_{+}$has only eigenvalues with positive real parts. A column decomposition of $E$ corresponding to the blocks of $\Lambda$ is denoted by $E=\left(E_{-}, E_{+}, E_{0}\right)$ and a row decomposition of $E^{-1}$ by

$$
E^{-1}=\left(\begin{array}{c}
E_{-}^{-1} \\
E_{+}^{-1} \\
E_{0}^{-1}
\end{array}\right) .
$$

The matrix $B$ has dimension $\left(n_{-}+n_{0}\right) \times n$ and $B\left(E_{-}, E_{0}\right)$ is nonsingular. These assumptions imply unique solvability of (4.20) (see Markowich [7]). We apply a collocation method to (4.20). In order to simplify the notation, we use the direct product of matrices, which for $X=\left(x_{i j}\right), i=1, \ldots, p, j=1, \ldots, q, Y=\left(y_{i j}\right)$, $i=1, \ldots, r, j=1, \ldots, s$ is defined as the $(p r \times q s)$-matrix

$$
X \otimes Y=\left(\begin{array}{cccc}
x_{11} Y & x_{12} Y & \cdots & x_{1 q} Y \\
x_{21} Y & & & \\
\vdots & & & \\
x_{p 1} Y & \ldots & \ldots & x_{p q} Y
\end{array}\right) .
$$

Denoting the components of $y$ by $y^{(l)}, l=1, \ldots, n$, we define $\hat{y}_{i}=$ $\left(y_{i 1}^{(1)}, y_{i 1}^{(2)}, \ldots, y_{i 1}^{(n)}, y_{i 2}^{(1)}, \ldots, y_{i k}^{(n)}\right)^{T}$. The collocation scheme then reads

$$
\begin{gathered}
\hat{y}_{i}=\left(1_{k} \otimes I_{n}\right) y_{i}+h_{i}(A \otimes M) \hat{y}_{i}+h_{i}\left(A \otimes I_{n}\right) \hat{f}_{i}, \\
y_{i+1}=y_{i}+h_{i}\left(b^{T} \otimes M\right) \hat{y}_{i}+h_{i}\left(b^{T} \otimes I_{n}\right) \hat{f}_{i}, \\
B y_{0}=\beta, \quad E_{+}^{-1} y_{N}=\gamma .
\end{gathered}
$$

We introduce the transformation

$$
y_{i j}=E\left(\begin{array}{c}
u_{i j} \\
v_{i j} \\
w_{i j}
\end{array}\right), \quad y_{i}=E\left(\begin{array}{c}
u_{i} \\
v_{i} \\
w_{i}
\end{array}\right)
$$

and obtain analogously to Subsection (a), and using the properties of the direct product (see Lancaster [6]),

$$
\begin{gathered}
u_{i+1}=\gamma\left(h_{i} \Lambda_{-}\right) u_{i}+h_{i} \Gamma\left(h_{i} \Lambda_{-}\right)\left(I_{k} \otimes E_{-}^{-1}\right) \hat{f}_{i}, \\
v_{i}=\gamma\left(-h_{i} \Lambda_{+}\right) v_{i+1}-h_{i} S\left[\Gamma\left(-h_{i} S\left(\Lambda_{+}\right)\right)\right]\left(I_{k} \otimes E_{+}^{-1}\right) \hat{f}_{i}, \\
w_{i+1}=w_{i}+h_{i}\left(b^{T} \otimes E_{0}^{-1}\right) \hat{f}_{i}, \\
B E\left(\begin{array}{c}
u_{0} \\
v_{0} \\
w_{0}
\end{array}\right)=\beta, \quad v_{N}=\gamma,
\end{gathered}
$$

where for an $l \times l$-matrix $Z$

$$
\begin{gathered}
\gamma(Z)=I_{l}+\left(b^{T} \otimes Z\right)\left(I_{l k}-(A \otimes Z)\right)^{-1}\left(1_{k} \otimes I_{l}\right), \\
\Gamma(Z)=\left(b^{T} \otimes I_{l}\right)\left(I_{l k}-(A \otimes Z)\right)^{-1}
\end{gathered}
$$


holds. Obvious changes in (4.7), (4.15) and (4.19) extend the definitions of $Y_{n, m}$, $H_{n, m}^{-}, H_{n, m}^{+}$and $H_{n, m}^{0}$ to functions of a matrix $P$ instead of a scalar $\lambda$. As in Markowich and Ringhofer [8], we use the equation

$$
\phi(P)=\frac{1}{2 \pi i} \int_{C} \phi(\lambda)(\lambda I-P)^{-1} d \lambda
$$

which holds for analytic matrix functions $\phi$. The contour $C$ encloses all eigenvalues of $P$. Since the eigenvalues of $\Lambda_{-}$and $\Lambda_{+}$are bounded away from zero, this device can be used to obtain stability estimates for (4.22), using the results of Subsection (a). A particular solution of (4.22) can be written as

$$
H_{I, i}(\Lambda, h) \hat{f}=\left(\begin{array}{c}
H_{I, i}^{-}\left(\Lambda_{-}, h\right) \hat{f} \\
H_{i, N}^{+}\left(\Lambda_{+}, h\right) \hat{f} \\
H_{I, i}^{0}(h) \hat{f}
\end{array}\right)
$$

and

$$
\left\|H_{I, \cdot}(\Lambda, h) \hat{f}\right\|_{L_{h}^{\infty}\left[t_{I}, t_{N}\right]} \leqslant \text { const }\|\hat{f}\|_{L_{h}^{1}\left[t_{l}, t_{N}\right]}
$$

holds.

Continuous dependence on $\beta$ and $\gamma$ is obvious, because of the structure of the boundary conditions in (4.22) and the assumption that $B E_{-}$is nonsingular. Thus, we have

$$
\|\hat{y}\|_{L_{h}^{\infty}\left[t_{0}, t_{N}\right]} \leqslant \operatorname{const}\left(\|\beta\|+\|\gamma\|+\|\hat{f}\|_{L_{h}^{1}\left[t_{0}, t_{N}\right]}\right)
$$

(c) Linear Systems. A problem of the form

$$
\begin{gathered}
y^{\prime}=\tilde{M}(t) y+f(t), \quad \text { with } \tilde{M}(t)=M+G(t), E^{-1} M E=\Lambda, \\
\|G(t)\| \leqslant \text { const } t^{-1-\nu}, \\
B y(0)=\beta, \quad E_{+}^{-1} y(T)=\gamma,
\end{gathered}
$$

is considered, where $B$ satisfies the assumptions (see Markowich [7]) guaranteeing unique solvability of (4.26). Applying a collocation method yields

$$
\begin{gathered}
\hat{y}_{i}=\left(1_{k} \otimes I_{n}\right) y_{i}+h_{i}(A \otimes M) \hat{y}_{i}+h_{i}\left(A \otimes I_{n}\right)\left(\hat{G}_{i} \hat{y}_{i}+\hat{f}_{i}\right), \\
y_{i+1}=y_{i}+h_{i}\left(b^{T} \otimes M\right) \hat{y}_{i}+h_{i}\left(b^{T} \otimes I_{n}\right)\left(\hat{G}_{i} \hat{y}_{i}+\hat{f}_{i}\right), \\
B y_{0}=\beta, \quad E_{+}^{-1} y_{N}=\gamma,
\end{gathered}
$$

where $\hat{G}_{i}=\operatorname{diag}\left(G\left(t_{i 1}\right), \ldots, G\left(t_{i k}\right)\right)$. A stability result for (4.27) is provided by the following

THEOREM 4.1. For some $\xi>0$, we define $\bar{h}$ by $\bar{h}=\max _{t_{i} \leqslant \xi} h_{i}$. If $\bar{h}$ is small enough and the condition $h_{i+1} \leqslant$ const $h_{i}$ holds for $t_{i}>\xi$, then (4.27) has a unique solution $\hat{y}$ which satisfies

$$
\|\hat{y}\|_{L_{h}^{\infty}\left[t_{0}, t_{N}\right]} \leqslant \text { const }\left(\|\beta\|+\|\gamma\|+\|\hat{f}\|_{L_{h}^{1}\left[t_{0}, t_{N}\right]}\right)
$$

Outline of the Proof. We consider the terms $\hat{G}_{i} \hat{y}_{i}$ in (4.27) as perturbations. Then a solution of (4.27) can be written as

$$
y_{i}=\bar{y}_{i}+E H_{I, i}(\Lambda, h) \hat{G} \hat{y},
$$


where $\bar{y}_{i}$ is a solution of the unperturbed problem and $\hat{G}$ is an appropriately defined matrix containing the $\hat{G}_{i}$ with $i \geqslant I$. Using (4.24), we obtain

$$
\begin{aligned}
\left\|E H_{I, i}(\Lambda, h) \hat{G} \hat{y}\right\|_{L_{h}^{\infty}\left[t_{I}, t_{N}\right]} & \leqslant \text { const }\|\hat{G} \hat{y}\|_{L_{h}^{1}\left[t_{I}, t_{N}\right]} \\
& \leqslant \operatorname{const}\|\hat{y}\|_{L_{h}^{\infty}\left[t_{I}, t_{N}\right]} \sum_{i=I}^{N-1} h_{i} t_{i}^{-1-\nu} \\
& \leqslant \operatorname{const}\|\hat{y}\|_{L_{h}^{\infty}\left[t_{I}, t_{N}\right]} \sum_{i=I}^{N-1} \frac{h_{i}}{h_{i-1}} h_{i-1} t_{i}^{-1-\nu} \\
& \leqslant \text { const }\|\hat{y}\|_{L_{h}^{\infty}\left[t_{I}, t_{N}\right]} \int_{t_{I}}^{t_{N}} t^{-1-\nu} d t \leqslant \text { const } t_{I}^{-\nu}\|\hat{y}\|_{L_{h}^{\infty}\left[t_{I}, t_{N}\right]} \\
& \leqslant \frac{1}{2}\|\hat{y}\|_{L_{h}^{\infty}\left[t_{t}, t_{N}\right]}
\end{aligned}
$$

if we choose $\xi=t_{I}$ big enough. The final result is now obtained as in Markowich and Ringhofer [8] or de Hoog and Weiss [5] by applying the standard theory for collocation methods on the interval $\left[t_{0}, t_{I}\right]$ and a contraction mapping argument on $\left[t_{I}, t_{N}\right]$.

5. Convergence. In this section we consider nonlinear problems of the form

$$
\begin{gathered}
x_{T}^{\prime}=f\left(t, x_{T}\right), \quad 0 \leqslant t \leqslant T(\varepsilon), \\
b\left(x_{T}(0)\right)=0, \\
E_{+}^{-1} x_{T}(T(\varepsilon))=0,
\end{gathered}
$$

with $T(\varepsilon)$ defined by $(2.2)$.

We apply a $k$-stage collocation method to (5.1), where $\omega(t)=\left(t-u_{1}\right) \cdots\left(t-u_{k}\right)$ satisfies the following assumptions

$$
\begin{gathered}
\int_{0}^{1} s^{p} \omega(s) d s=0, \quad p=0, \ldots, r-1, \\
\int_{0}^{1} s^{r} \omega(s) d s \neq 0 .
\end{gathered}
$$

For an arbitrary set of collocation points $0 \leqslant u_{1}<\cdots<u_{k} \leqslant 1, r=0$ holds. For Gauss points, $r=k$, and for Lobatto points, $r=k-2$.

The main result of this paper is stated in

THEOREM 5.1. Let the assumptions of Theorem 2.1 be valid. Assume that $f$ is $(k+r)$-times differentiable with respect to both variables, the collocation scheme satisfies (5.2), and the mesh is defined by

$$
\begin{gathered}
h_{i}=c_{0} \varepsilon^{1 /(k+r)}, \quad 0 \leqslant t_{i} \leqslant \gamma, \\
h_{i}=\varepsilon^{1 /(k+r)} \exp \left(\frac{\lambda_{\min }-\delta}{k+r} t_{i}\right), \quad \gamma<t_{i}<T(\varepsilon),
\end{gathered}
$$

where $\delta>0$ and $\lambda_{\min }$ is defined in Section 2. Then, for $\varepsilon$ small enough, the collocation scheme for problem (5.1) has a solution $\hat{y}$ which is unique in a neighborhood of the solution $x_{T}$ of (5.1), which does not shrink as $\varepsilon \rightarrow 0$. $\hat{y}$ satisfies

$$
\begin{gathered}
\max _{0 \leqslant i \leqslant N}\left\|y_{i}-x_{T}\left(t_{i}\right)\right\|=O(\varepsilon), \\
\max _{\substack{0 \leqslant i \leqslant N \\
1 \leqslant j \leqslant k}}\left\|y_{i j}-x_{T}\left(t_{i j}\right)\right\|=O\left(\varepsilon^{(k+1) /(k+r)}\right) . \\
.
\end{gathered}
$$


Outline of the Proof. We introduce

$$
e_{i}=x_{T}\left(t_{i}\right)-y_{i}, \quad e_{i j}=x_{T}\left(t_{i j}\right)-y_{i j} .
$$

Linearization of the collocation scheme for the $e_{i}, e_{i j}$ and elimination of the $e_{i j}$ yield equations of the form

$$
\hat{e}_{i+1}=\gamma\left(h_{i} f_{y}\left(t_{i}, x_{T}\left(t_{i}\right)\right)\right) \hat{e}_{i}+l_{i} .
$$

From Axelsson [3] or Weiss [9], we obtain

$$
\left\|l_{i}\right\| \leqslant h_{i}^{k+r}\left\|x_{T}^{(k+r+1)}\right\|_{\left[t_{i}, t_{i+1}\right]} .
$$

The exponential decay of $x_{T}^{(k+r+1)}$, and our choice of the mesh, imply

$$
\begin{gathered}
\left\|l_{i}\right\|=O(\varepsilon), \quad 0 \leqslant t_{i} \leqslant \xi, \\
\left\|l_{i}\right\|=O\left(\varepsilon \exp \left(-\delta t_{i}\right)\right), \quad \xi<t_{i}<T(\varepsilon) .
\end{gathered}
$$

The assumptions on the mesh of Theorem 4.1 are satisfied for $\varepsilon$ small enough, because for $t_{i}>\xi$,

$$
\frac{h_{i+1}}{h_{i}}=\exp \left(\frac{\lambda_{\min }-\delta}{k+r} h_{i}\right) \leqslant \text { const }
$$

if $h_{i} \leqslant$ const. This follows from

$$
h_{N}=\varepsilon^{1 /(k+r)} \exp \left(\frac{\lambda_{\min }-\delta}{k+r} T(\varepsilon)\right) \leqslant \varepsilon^{1 /(k+r)} \exp \left(\frac{\lambda_{\min }}{k+r} \frac{\ln (1 / \varepsilon)}{\lambda_{\min }}\right)=1 .
$$

The estimates obtained in Section 4 can now be employed to apply a contraction mapping argument, implying locally unique solvability of the collocation scheme and

$$
\begin{aligned}
\max _{i=0, \ldots, N}\left\|e_{i}\right\| & \leqslant \text { const } \sum_{i=0}^{N-1} h_{i}\left\|l_{i}\right\| \leqslant \text { const }\left[\gamma \varepsilon+\varepsilon \sum_{t_{i}>\gamma} h_{i} e^{-\delta t_{i}}\right] \\
& \leqslant \operatorname{const}\left[\gamma \varepsilon+\varepsilon \sum_{t_{i}>\gamma} h_{i-1} e^{-\delta t_{i}}\right] \leqslant \operatorname{const} \varepsilon\left[\gamma+\int_{\gamma}^{T(\varepsilon)} e^{-\delta t} d t\right] .
\end{aligned}
$$

It is a well-known fact that the superconvergence order is only given at meshpoints. This explains the weaker result (5.4b) at collocation points.

Combining Theorems 2.1 and 5.1 shows that the collocation solution is an approximation of the solution $y^{*}$ of the infinite problem (1.1) and

$$
\max _{0 \leqslant i \leqslant N}\left\|y_{i}-y^{*}\left(t_{i}\right)\right\|=O(\varepsilon)
$$

holds. As in Markowich and Ringhofer [8], it can be shown that the necessary number of steps $N(\varepsilon)$ satisfies

$$
N(\varepsilon)=O\left(\varepsilon^{-1 /(k+r)}\right) .
$$

Institut für Angewandte und Numerische Mathematik

Technische Universität Wien

Wiedner Haupstrasse 6-10

A-1040 Wien, Austria 
1. U. AsCheR \& R. Weiss, "Collocation for singular perturbation problems I: First order systems with constant coefficients," SIAM J. Numer. Anal., v. 20, 1983, No. 3, pp. 537-557.

2. U. ASCHER \& R. WEISs, "Collocation for singular perturbation problems III: Nonlinear problems without turning points," SIAM J. Sci. Statist. Comput., v. 5, 1984, No. 4, pp. 811-829.

3. O. AXelsSON, “A class of $A$-stable methods," $B I T$, v. 9, 1969, pp. 185-199.

4. C. DE Boor \& B. SwARTZ, “Collocation at Gaussian points," SIAM J. Numer. Anal., v. 10, 1973, pp. 582-606.

5. F. R. DE Hoog \& R. Weiss, “An approximation method for boundary value problems on infinite intervals," Computing, v. 24, 1980, pp. 227-239.

6. P. Lancaster, Theory of Matrices, Academic Press, New York, 1969.

7. P. A. MARKowich, "A theory for the approximation of solutions of boundary value problems on infinite intervals,” SIAM J. Math. Anal., v. 13, 1982, pp. 484-513.

8. P. A. MaRKowich \& C. A. Ringhofer, "Collocation methods for boundary value problems on 'long' intervals," Math. Comp., v. 40, 1983, pp. 123-150.

9. R. Weiss, "The application of implicit Runge-Kutta and collocation methods to boundary value problems," Math. Comp., v. 28, 1974, pp. 449-464.

10. K. Wright, "Some relationships between implicit Runge-Kutta, collocation and Lanczos $\tau$ methods, and their stability properties," $B I T$, v. 10,1970 , pp. 217-227. 